\title{
ESTUDIO COMPARATIVO DE LAS POLÍTICAS PÚBLICAS EN COMPETITIVIDAD A NIVEL CENTROAMERICANO 1
}

\author{
Andrea Vanessa Espinoza Martínez ${ }^{2}$ \\ Facultad de Ciencias Sociales y Administrativas \\ Universidad Tecnológica Centroamericana, San Pedro Sula, Honduras
}

(Enviado: Septiembre, 2017/ Aceptado: Agosto, 2018)

\begin{abstract}
Resumen
El estudio de las políticas públicas no es algo nuevo. Desde el año 1960 se ha desarrollado un creciente interés por el estudio de las políticas públicas, con enfoque en las ciencias sociales y en especial en la ciencia política. Las políticas públicas representan una estrategia para la administración del Estado y permiten espacios de diálogo entre el sector privado, gubernamentales y sociedad civil. Los temas que abarcan las políticas públicas son variados. Sin embargo, uno de los de mayor preocupación para los gobiernos en la actualidad es la competitividad, pues la competencia internacional se hace cada vez más difícil y los Estados deben tener un valor agregado que permita su protagonismo en el escenario internacional. Entonces, las políticas públicas se convierten en parte de una estrategia de competitividad por parte de los estados. Siendo Centroamérica, una región tan amplia como diversa, contar con políticas públicas en materia de competitividad permite concentrarse en el cumplimiento de los doce pilares del índice de Competitividad Global y obtener una mejor reputación tanto a nivel de países como de región.
\end{abstract}

Palabras Claves: Políticas Públicas, Competitividad, Ciencias Políticas

\begin{abstract}
The study of the public policy is not something new. Since 1960 the interest for studying public policy has focused in the frame of the social sciences and specifically in political science. The public policies represent a strategy for the administration of State and allow dialogue opportunities between the private sector, governmental sector and civil society. The topics covered by public policies are varied. However, the concern for international competitiveness is due to the fact that it is increasingly difficult, and states need to have an added value that allows their prominence in the international scenario. Then, public policies become part of a strategy of competitiveness on the part of states. Since Central America is a region as wide as diverse, having public policies on competitiveness allows us it focus on compliance with the twelve levels of competitiveness of globalization, and that would help obtain a better reputation both at the level of countries such as region.
\end{abstract}

Key Words: Public Policy, Competitiveness, Political Science

\footnotetext{
${ }^{1}$ Este artículo se desprende del proyecto de investigación presentado como requisito previo a la obtención del título de Licenciada en Relaciones Internacionales. Fue elaborado en el marco del Observatorio de Competitividad del InnovaLab. Asesora Temática y Metodológica: Dra. Claudia María Castro Valle.

${ }^{2}$ Autora para correspondencia. Email: andreavanessa96@hotmail.com
} 


\section{Introducción}

La interdependencia estatal como la competencia internacional ha llevado a que cada Estado implemente estrategias para solventar problemas que perjudican a la sociedad como también lograr estabilidad y desarrollo tanto económico como humano. Las políticas públicas se han constituido como esos vehículos del crecimiento y del desarrollo; son estas políticas el mecanismo idóneo para que los gobiernos aborden temas de interés común, ya que son multidisciplinarias que abarcan aspectos que tienen que ver con la educación, salud, pobreza, economía entre otros.

En resumen, se podría decir que las políticas públicas constituyen la definición de objetivos que una sociedad quiere perseguir, esas líneas de acción que se deben de seguir y lo instrumentos necesarios para lograrlos. Dentro de los temas que las políticas públicas también trabajan, es en cuanto a competitividad. La competitividad son aquellos elementos como, recursos, territorio, ubicación como niveles políticos, económicos y sociales que ocasionan que el país tenga una ventaja competitiva que permita que se destaque dentro del resto de países.

Para ello el Foro Económico Mundial, realiza un estudio de la competitividad de ciento cuarenta y dos países por medio del índice de Competitividad Global, el cual contempla doce pilares: Instituciones, Infraestructura, Entorno Macroeconómico, Salud y Educación Primaria, Educación Superior y Capacitación, Eficiencia de los Mercados de Bienes, Eficiencia del Mercado Laboral, Desarrollo del Mercado Financiero, Preparación Tecnológico, Tamaño del mercado, Sofisticación de los Negocios e Innovación.

En cuanto a las políticas públicas en materia de competitividad, los países centroamericanos cuentan con dichas políticas ya sea directamente o por medio de programas y planes tanto a corto, mediano y largo plazo. Sin embargo, no todos los países de Centroamérica contemplan políticas públicas específicas en materia de competitividad, pero sí para cada uno de los pilares que dicta el Foro Económico Mundial que indirectamente contribuyen a crear ese espacio idóneo para que el país sea más competitivo y las políticas públicas creadas sean del todo inclusivas y lograr el bien común siendo este el principal objetivo del Estado.

\subsection{Objetivo general}

Realizar un análisis comparativo de las políticas públicas en Centroamérica orientadas a la competitividad

\subsection{Objetivos específicos}

a. Determinar la relevancia de las políticas públicas para el gobierno de un Estado.

b. Determinar si la competitividad representa un aspecto prioritario para los países centroamericanos.

c. Investigar los diferentes avances en competitividad de Centroamérica.

d. Determinar si los avances obtenidos en políticas públicas en Centroamérica han sido competitivos. 


\section{Marco teórico}

\subsection{Definición de política pública}

Los países en la actualidad utilizan las políticas públicas como medida de un buen accionar del gobierno de turno como también solventar problemas que acontecen en la sociedad en general. Existen muchas definiciones de políticas públicas, siendo una de las más completas en su definición la propuesta por Peters (1982), quien señala que un política pública es “el conjunto de actividades de las instituciones de gobierno, actuando directamente o a través de agentes, y que van dirigidas a tener una influencia determinada sobre la vida de los ciudadanos”.

Esta definición enfatiza la importancia de la participación gubernamental en la formulación de las mismas para poder lograr un impacto que transforme la vida de los ciudadanos. Las políticas públicas se han convertido en ese puente de comunicación entre la sociedad civil, gobierno y entes privados para brindar solución a un problema común (Almond, 1978).

(Lahera, 2002) afirma:

"Una política pública de excelencia aquella que corresponde a aquellos cursos de acción y flujos de información relacionados con un objetivo político definido en forma democrática; los que son desarrollados por el sector público y, frecuentemente, con la participación de la comunidad y el sector privado. Una política pública de calidad incluirá orientaciones o contenidos, instrumentos o mecanismos, definiciones o modificaciones institucionales, y la previsión de sus resultados”.

Si no existen medios tanto de inclusión como instrumentos de medición de las políticas la formulación e implementación de las mismas sería en vano.

\subsection{Características de las políticas públicas}

Al ser un instrumento de accionar tanto del gobierno como demás actores que conforman el Estado, las políticas públicas cuentan con objetivos públicos que se buscan alcanzar en el corto y largo plazo como cambios sociales definidos en forma democrática como menciona (Pereyra, 2007). Estos instrumentos procuran mejorar la calidad de vida de la gente, garantizar sus derechos y optimizar sus servicios públicos.

Según el estudio las características que debe contener una política pública son las siguientes (Pereyra, 2007) afirma:

1. Tienen diferentes orígenes: la agenda pública, la propuesta de sectores específicos o la concertación de diferentes actores.

2. Implican en corresponsabilidad a los diferentes actores en todo el ciclo de su gestión: formulación y decisión, definición del presupuesto y fuentes de financiamiento, ejecución y control.

3. Indican cómo se implementará, quiénes serán los responsables de su ejecución, cuáles serán los mecanismos de participación ciudadana y cómo se rendirán cuentas de sus logros obtenidos; garantizan un flujo de información entre los ciudadanos y las instituciones que las diseñan e implementan.

4. Cuentan con instrumentos específicos para hacer efectiva su implementación, sin quedarse en generalidades y buenas intenciones. 
Otra de las características de las cuales es necesario conocer y valorar es la relación existente entre las políticas públicas y dos de los actores implicados como el Estado y Sociedad (Mendíaz, 2004). Mendíaz afirma que: "Una parte fundamental del quehacer del gobierno se refiere al diseño, gestión y evaluación de las políticas públicas”. La respuesta del porqué las políticas públicas permiten una mejor relación entre Estado y la sociedad se resumen en los siguientes puntos:

1. Las políticas públicas son soluciones específicas de cómo manejar los asuntos públicos.

2. Se desprenden de la agenda pública.

3. Son habitualmente una mejor opción respecto a una política óptima en lo sustantivo.

4. El gobierno puede ser concebido como un instrumento para la realización de las políticas públicas, siendo el foco del gobierno estar en sus resultados.

5. El desafío de dar continuidad y a la vez de innovar en las políticas públicas como discontinuidad de alianzas y política

Tal como lo decía Robert Dewey (uno de los autores precursores del estudio de las políticas públicas como disciplina), las políticas públicas se vuelven necesarias para desarrollar un vínculo entre la "resolución experta de problemas con una sociedad democrática activa" (Parada, 2002).

\subsubsection{Diferencia entre política pública, programa y ley}

Al ser un tema de estudio relativamente reciente, en muchas ocasiones su concepto etimológico como la razón de ser de dichas políticas se llega a distorsionar. Es por ello que muchos estudiosos se han encargado de la tarea de brindar aclaraciones haciendo la diferencia entre política pública, programa y ley de una manera más amplia. En diversas ocasiones se llega a confundir la política pública con un programa público (Fernández Arroyo, 2012).

La diferencia entre ambas radica principalmente en que un programa es una intervención pública directa sobre una realidad social, que se usa, en conjunto con otros programas (y otros instrumentos de política), para implementar una política. Es decir que dentro de un programa van inmersas en las ciertas políticas que son necesarias para el buen manejo de los objetivos de los Estados, lo cual es la constante diferencia entre ley.

En cuanto a las leyes, y las políticas públicas, las primeras son un componente formal de una política pública, no la política pública en sí. Dada la importancia que tienen las normas formales al interior de un arreglo institucional, son comúnmente empleadas para sellar un acuerdo alrededor de un asunto de política (Torres Melo \& Santander, 2013). La ley es un mecanismo fundamental para garantizar que la decisión política que se tomó se mantenga y guíe el curso de acción de la política pública.

\subsection{La relación entre políticas públicas y democracia}

El término democracia es demasiado amplio y parar poder desarrollarse a cabalidad se necesita la participación por parte del pueblo en cualquiera de los temas ya sea económico, político o social. De igual forma, la incertidumbre que genera la posibilidad de que agentes estén interesados en desviar la trayectoria de la estrategia lleva a que los hacedores de política empleen artefactos institucionales, como las normas y las leyes, para hacer de obligatorio cumplimiento la decisión de política (Gault \& Blanco, 2013).

En democracia, por tanto, las políticas públicas han de ser necesariamente para ciudadanos; esto es, las políticas públicas han de permitir a los individuos ejercer su papel de ciudadanos. Y puesto que ser ciudadano es poder participar de la vida política los asuntos de las polis, una política para ciudadanos es aquélla que ha nacido de la acción política, del diálogo público, de 
la participación en la vida pública que define a la ciudadanía (Canto Sáenz, 2014). Entonces estaríamos hablando en verdad del gobierno del pueblo, para el pueblo. Si bien es cierto la democracia representa un aspecto fundamental en cuanto a políticas pública, debido que estas contienen un proceso bien estratificado para poder realizarse.

\subsection{Políticas públicas en Centroamérica}

Es importante considerar las acepciones que tienen las políticas públicas por parte del territorio centroamericano, el cual ha estado sujeto a varios problemas que estas políticas representan esa luz que guiará el camino para un mejor panorama en la administración estatal. Recordando la importancia de las mismas, las políticas públicas son acciones ejecutadas para responder a las distintas demandas sociales, de salud, educación, empleo y desarrollo social (Monge Pacheco, 2014). Se considera fundamental la participación ciudadana en los procesos de establecer políticas públicas porque constituye un elemento fundamental y una condición para propiciar la gobernabilidad democrática, y puede llegar a constituir un mecanismo para el empoderamiento social.

"Las Políticas Públicas tienen la potencialidad de resolver problemas concretos, de promover integración social, es decir, permitir que la gente viaje en el mismo avión.” (Delgado Godoy, 2009). En el "mismo avión” hace referencia a la búsqueda de la equidad, ya que una propuesta de política puede beneficiar a unos y perjudicar a otros, se debe tener claro que la mayoría es quien decide en una democracia, sin dejar a un lado a las minorías.

De acuerdo con lo anterior, al observar o analizar el marco jurídico existente en la región centroamericana se debe tener presente que si bien, en muchos casos, existe legislación que de alguna forma regula la producción y el uso, acceso y conservación de los recursos naturales, generalmente no se da o ha dado, la correspondiente institucionalidad de la norma y en aquellos casos que sí se ha dado, es común la inexistencia de la respectiva cultura jurídica que dé fundamento a su efectiva aplicación (Chacón \& Lawrence, 1996). En este sentido, tradicionalmente los países centroamericanos se han caracterizado por haber legislado y normado para regular casi toda actividad que se da en cada país.

La relevancia de su actuación, en este campo, del medio ambiente y administración de recursos, ha variado dependiendo, en gran medida, de los aires internacionales y del gobierno de turno. Por otra parte, si bien en los países de la región se puede hablar de la existencia de un mosaico de legislación, en general esta no ha nacido de una política coherente del gobierno.

Además, es importante resaltar que en los países centroamericanos no han existido políticas públicas coherentes que hayan venido a buscar la sostenibilidad tanto ambiental como económica de cada país. En cada momento histórico, generalmente han existido decisiones casuísticas y de corto plazo, que han contradicho decisiones anteriores, contemporáneas o futuras. Como ejemplo de esto, es común que las políticas de un Ministerio o Secretaría de Estado sean contradictorias con lo ejecutado o previsto por otro.

Así mismo, es importante recordar que históricamente se consideró que un mayor tamaño estatal proveería el marco institucional necesario para el desarrollo económico y social de la región. Por ello, en décadas pasadas, se crearon múltiples entes públicos que vinieron a tener un papel muy importante en la producción de cada país, así como en la conservación de sus recursos naturales (Chacón \& Lawrence, 1996).

\subsection{Competitividad}

Para comprender la importancia que representa poder contar con políticas públicas en materia de competitividad, se debe conocer el amplio concepto de la competitividad. Considerando el 
término anteriormente mencionado como "la capacidad de una organización para obtener y mantener sistemáticamente unas ventajas comparativas que le permiten alcanzar, sostener y mejorar una determinada posición en el entorno socioeconómico en la actualidad” (Mendoza Félix, 2015).

Sin embargo, otros autores utilizan una definición un poco más en términos económicos y productivos, Siendo "la capacidad de un país de exportar y vender en los mercados externos como su capacidad de defender su propio mercado doméstico respecto a una excesiva penetración de importaciones” (Bejarano, 1997). El ente encargado a nivel internacional ha sido el Foro Económico Mundial el cual determinó 12 Pilares que permiten poder medir el grado de competitividad de un país como su análisis en comparación en un ranking de 142 países (Chapa Gonzáles, 2010). Siendo autores como Villareal quien plantea:

“que la competitividad va más allá de la productividad, representa un proceso centrado en generar y fortalecer las capacidades productivas y organizacionales para enfrentar de manera exitosa los cambios del entorno, transformando las ventajas comparativas en competitivas, dándole sustentabilidad a través del tiempo como condición indispensable para alcanzar niveles de desarrollo elevados.” (Quero, 2008).

Entonces, dicha definición sustenta lo establecido en los 12 pilares de competitividad, dentro de los cuales se encuentran:

1. Instituciones

2. Infraestructura

3. Entorno macroeconómico

4. Salud y educación primaria

5. Educación superior y capacitación

6. Eficiencia de los mercados de bienes

7. Eficiencia del mercado laboral

8. Desarrollo del mercado financiero

9. Preparación tecnológica

10. Tamaño del mercado

11. Sofisticación de los negocios

12. Innovación.

En cuanto a la competitividad actual un término ligado a la misma es el auge de la competitividad sistémica. El modelo sistémico de la competitividad viene a reconocer de facto que el logro de un nivel de desarrollo industrial-económico realista, no puede alcanzarse o mantenerse solo a base de condiciones macroeconómicas estables en el nivel macro, o de condiciones específicas de producción en el nivel micro (Calderón Vásquez, 2015).

Asimismo, "la capacidad de integrar las políticas micro y macro depende de la existencia de toda una serie de estructuras políticas y económicas y de un conjunto de factores socioculturales y patrones básicos de organización a nivel meta” (Vázquez, 2016).Por lo tanto, la competitividad a nivel territorial es el producto de un entorno donde se crean y mantienen las condiciones para que el esfuerzo competitivo se produzca, entorno que no puede improvisarse sino construirse.

El Estado y las políticas públicas por él operadas, desempeñan un papel decisivo en la definición del proceso de desarrollo, su consolidación o la reestructuración productiva de una economía, especialmente bajo las nuevas modalidades de gestión gubernamental. Es por eso que si los países contienen políticas públicas de calidad pueden empoderarse en el tema competitivo y ser atractivos para el resto de países de la comunidad internacional (Plataformas para Informacion 
para Politicas Públicas, 2013). A medida que se incrementa la complejidad de nuevas formas de organización industrial, la naturaleza integral de la competitividad adquiere más importancia, aunque ello no sea igualmente relevante para todo tipo de actividad económica.

\section{Metodología}

La metodología utilizada para la investigación es mediante un enfoque cualitativo siendo una investigación descriptiva y exploratoria (Malhotra, 2008). Dicho enfoque es cualitativo, ya que se obtendrán información por medio de datos primarios y de datos secundarios con el objetivo de realizar un análisis comparativo de las políticas públicas en Centroamérica orientada a la competitividad. El problema de investigación definido para realizar la investigación es el siguiente: ¿Hay diferencias con relación a las políticas públicas en competitividad en Centroamérica, dados los resultados tan dispares obtenidos por estos países en los rankings internacionales? Para fundamentar el estudio se ha hecho uso de ciertas variables de investigación y de hipótesis, siendo utilizadas para poder obtener el registro de las políticas públicas estudiadas (Tabla 1).

Hipótesis: Las diferencias son mayores que las similitudes en las políticas públicas formuladas en materia de competitividad disponibles en los diferentes Estados centroamericanos.

Tabla 1. Variables de Investigación en el estudio de Políticas Públicas

\begin{tabular}{|c|c|c|}
\hline Variables & Definiciones & Operatividad \\
\hline $\begin{array}{l}\text { Variable } \\
\text { independiente: } \\
\text { Políticas Públicas en } \\
\text { materia de } \\
\text { competitividad } \\
\text { formuladas en los } \\
\text { Estados } \\
\text { Centroamericanos }\end{array}$ & $\begin{array}{l}\text { Políticas públicas son aquellas gestiones del gobierno } \\
\text { junto con entes privados y la sociedad civil con el } \\
\text { objetivo de solventar un problema de orden público y } \\
\text { lograr una mejor calidad de vida para los ciudadanos. } \\
\text { Su formulación es en base a } 4 \text { fases primordiales: } \\
\text {-Análisis del problema } \\
\text {-Análisis de soluciones } \\
\text {-Análisis de factibilidad } \\
\text {-Plan de acción de política pública }\end{array}$ & $\begin{array}{l}\text { Políticas públicas publicadas en } \\
\text { materia de competitividad } \\
\text { vigentes en Centroamérica, } \\
\text { publicadas en canales oficiales. }\end{array}$ \\
\hline $\begin{array}{l}\text { Variable dependiente: } \\
\\
\text { Diferencias y } \\
\text { similitudes } \\
\text { identificadas en las } \\
\text { Políticas Públicas } \\
\text { disponibles en } \\
\text { Centroamérica en } \\
\text { materia de } \\
\text { competitividad }\end{array}$ & $\begin{array}{l}\text { Las similitudes en cuanto a políticas públicas en } \\
\text { materia de competitividad se guían en base a los } 12 \\
\text { pilares de competitividad: } \\
\text {-Entorno Institucionalidad } \\
\text {-La infraestructura amplia y eficiente } \\
\text {-Estabilidad Macroeconómico } \\
\text {-Salud y Educación Primaria } \\
\text {-Educación superior } \\
\text {-Eficiencia de mercado de mercancías } \\
\text {-Eficiencia de mercado laboral } \\
\text {-Sofisticación del mercado financiero } \\
\text {-Disposición tecnológica } \\
\text {-Tamaño de mercado } \\
\text {-Sofisticación de los negocios } \\
\text {-Innovación }\end{array}$ & $\begin{array}{l}\text { Análisis comparativo de las } \\
\text { políticas públicas en materia de } \\
\text { competitividad vigentes en } \\
\text { Centroamérica. }\end{array}$ \\
\hline & $\begin{array}{l}\text { Por medio de estos } 12 \text { pilares se identificarán las } \\
\text { características comunes de las políticas públicas } \\
\text { centroamericanas en materia de competitividad. }\end{array}$ & \\
\hline
\end{tabular}

Fuente: Elaboración propia con datos obtenidos de Quero, L. (2008). Estrategias Competitivas de la Revista Científica Electrónica Ciencias Gerenciales (NEGOTIUM), 1-14. 
En el estudio de la política pública se ha obtenido la información por medio de fuentes primaria propias para dicha investigación como ser libros, revistas científicas, y de investigación de instituciones públicas o privadas y entrevistas de profundidad a autoridades de la Cámara de Comercio de Cortes y el Salvador. Así mismo, a encargados del área de Competitividad de la nación, del Ministerio de Planificación Nacional y Política Económica (MIDEPLAN) y Transporte y Logística del SIECA. Mientras que las fuentes secundarias de información se ha hecho uso de documentos oficiales de instituciones públicas y privadas, informes técnicos que contienen información organizada en relación con el estudio.

\section{Resultados}

Como descrito en los párrafos anteriores, las políticas públicas son aquellas acciones donde la sociedad civil, entes privados y el gobierno interactúan para poder solucionar un problema común. Si se analiza la Región Centroamericana, las asimetrías propias de estos países hace que se dificulte considerar o pensar que puedan elaborarse verdaderas políticas públicas. Sin embargo, desde una perspectiva más general, en un contexto como éste, las políticas públicas serían ese espacio de diálogo que permita que la sociedad civil tenga voz, que los entes que conforman el Estado consideren que trabajando en conjunto es como se daría solución a un problema común.

Un aspecto importante en la administración de las políticas públicas es la regulación de las mismas. Es decir, el ente encargado de tener documentadas dichas políticas, como el centro donde se puedan encontrar de una manera más rápida. La Tabla 2 muestra la lista de entidades a cargo de las políticas públicas en Centroamérica.

Tabla 2. Entidades que contemplan las políticas públicas en Centroamérica

\begin{tabular}{ll}
\hline \multicolumn{1}{c}{ País } & \multicolumn{1}{c}{ Entidad que contempla las políticas públicas } \\
\hline Honduras & Secretaría de Coordinación General de Gobierno (Dirección Presidencial de \\
& Planificación Estratégica, Presupuesto, Inversión Pública y Cooperación Externa) \\
Guatemala & Secretaría de Planificación y Programación (SEGEPLAN) \\
El Salvador & Secretaría Técnica de Planificación (SETEPLAN) \\
Costa Rica & Ministerio de Planificación Nacional y Política Económica \\
Nicaragua & Ministerio de Gobernación/Instituto de Estudios estratégico y políticas publicas \\
Panamá & Secretaría de Comunicación del Estado \\
Belice & Ministry of Labor, Local Government and Rural Development, Public Service, \\
& Energy \& Public Utilities \\
\hline
\end{tabular}

Fuente: Elaboración propia con datos obtenidos de Secretaria de Comunicación del Estado, 2017; Secretaria General de Despacho de Gobierno, 2016; Secretaria de Planificación y Progrmacion Republica de Guatemala; Secretaria Tecnica y de Planificación Republica de El Salvador, 2014;Ministerio de Planificación y Política Económica,2016; Ministerio de Gobernación 2016; Belize Ministry of Labor, Local Government and Rural Development, Public Service, Energy \& Public Utilities, 2017

Por ejemplo, en Honduras, la Dirección Presidencial de Planificación Estratégica, Presupuesto e Inversión Pública es la que se encarga de promover políticas globales y sectoriales para la promoción de programas gubernamentales, como establecer políticas de igual y equidad social, en fin, esta es la que publica las políticas públicas vigentes. En comparación con el resto de países de la región, Guatemala cuenta con políticas públicas incluso históricas y que han servido 
de base e inspiración para la formulación como la implementación de las mismas (Secretaria General de Despacho de Gobierno, 2016).

Guatemala cuenta con un Centro de Acopio de Políticas Públicas donde se detalla aproximadamente 50 políticas públicas en diversos temas, sobre todo que Guatemala ha reconocido la importancia de las mismas, pero aun insiste que persisten problemas en cuanto al fortalecimiento de alianzas público-privadas para que se puedan realizar las políticas públicas en su totalidad (SEGEPLAN, 2016).

No se puede obviar a Costa Rica, siendo uno de los países centroamericanos donde la transparencia como también su institucionalidad fuerte han sido ejemplo para el istmo, las políticas públicas siempre han trabajado de la mano con las políticas públicas, para la administración de estas se encarga el Ministerio de Planificación , en el cual, como ellos lo describen en muchos de sus informes sobre estas políticas, este Ministerio es el que realiza un diagnostico en cuanto a la administración pública y los programas y demás herramientas que utilice para poder lograr el desarrollo de las políticas determinadas (MiDEPLAN, 2016).

El Salvador, puede ser pequeño en extensión territorial, pero de igual forma que el resto de los países centroamericanos cuenta con políticas públicas en temas como economía, política, salud, educación y diversos temas contemplados en su mayoría por la Secretaría Técnica de Planificación. Nicaragua por su parte, en el Ministerio de Gobierno como en el Instituto de Políticas Públicas, se han concentrado en diversos aspectos en cuanto a políticas públicas siempre tomando como principal factor el tema social, resaltan políticas como Derechos Humanos, Salud, Educación y Seguridad Ciudadana (SETEPLAN, 2014).

Un detalle en particular que cuenta Nicaragua, es que, aunque tenga pocas políticas públicas, si las aplican y evalúan constantemente, cosa que el resto de países centroamericanos se han quedado un poco atrás por no presentar avances de las políticas como los logros obtenidos o cuando concluyen las políticas. Un aspecto curioso es en cuanto a Panamá, donde no contemplan una unidad en específico que se encargue del manejo de las políticas públicas, es su mayoría es el Ministerio de Finanzas y Economía, como también la Secretaría de Gobierno la que proyecta ciertas políticas públicas, donde concuerda con Nicaragua, los temas van más enfocados en aspectos sociales y de fortalecimiento institucional (Ministerio de Gobernación, 2016).

Finalmente, Belice, considerándolo como un caso aparte comparado con el resto de los países de la región, no solo por el hecho de las diferencias lingüísticas, sino que cuenta con un Ministerio de Trabajo, Gobierno Local Servicios públicos y Políticas (Ministry of Labor, Local Government and Rural Development, Public Service, Energy \& Public Utilities), la mayoría de políticas públicas por formar parte del Commonlaw, toman aspectos similares a los países que tiene este sistema, centrándose en temas de mayor importancia como política, derechos humanos, educación y salud específicamente siempre con miras a considerarse como un país diferente al resto de países centroamericanos (Belize Ministry of Labor, 2017).

Analizando lo anteriormente mencionado, se pueden considerar como las más activas en el manejo de las políticas públicas a 4 instancias: SEGEPLAN ${ }^{3}$, MIDEPLAN $^{4}$, Dirección de Planificación Estratégica de Honduras ${ }^{5}$ y SETEPLAN ${ }^{6}$. El porqué de ello, y sin intención de

\footnotetext{
${ }^{3}$ Secretaría de Planificación y Programación

${ }^{4}$ Ministerio de Planificación Nacional y Política Económica

${ }^{5}$ Secretaría de Coordinación General de Gobierno

${ }^{6}$ Secretaría de Coordinación General de Gobierno
} 
crear discriminación con el resto de los países de la región, son estos países en los que se puede considerar que cuentan con una unidad específica en el tema de las políticas públicas.

Por ejemplo, SEGEPLAN en Guatemala tiene la Sub Secretaria de Políticas Públicas, convirtiéndose este en el espacio desde el cuál se brinda orientación técnica y metodológica para todos los aspectos del ciclo de políticas (diseño, implementación, seguimiento y evaluación).Incluso dentro de su página especifican que políticas públicas están vigentes por tema, convirtiéndose SEGEPLAN de vital importancia siendo fiel participe en los procesos de formulación de política, los cuáles ha plasmado, mediante un trabajo colectivo, participativo y académico, en la Guía para Formulación de Políticas Públicas y del Centro de Acopio de Políticas Públicas, entre otras instancias.

Lo anterior es un aspecto en común que tiene con Honduras, que particularmente luego de haber sido Secretaría de Planificación, se convierte en una Dirección de Planificación Estratégica y Cooperación para el año 2014, delegándole a esta las funciones principales de ser partícipes en la planificación estratégica nacional en el marco de Visión de País, ser esa instancia en la cual se propongan políticas globales generales y sectoriales para mejorar el impacto y eficacia de los programas gubernamentales, promover políticas de igualdad, en fin, crear un espacio idóneo donde se proyecten las políticas públicas.

De igual forma, Costa Rica, cuenta con MIDEPLAN, siendo ese órgano asesor y de apoyo técnico de la Presidencia de la República y su principal labora radica en formular, coordinar como también dar seguimiento y evaluar las estrategias y prioridades del Gobierno, en pocas palabras MIDEPLAN para Costa Rica le permite definir la visión, metas tanto a mediano como a largo plazo para un mejor desempeño del poder Ejecutivo primordialmente. La instancia encargada de formular, coordinar, dar seguimiento y evaluar las estrategias y prioridades del Gobierno; es decir, define la visión y metas de mediano y largo plazo que inspiran el accionar del Ejecutivo. También el Salvador cuenta con una Secretaría Técnica que se subdivide en tres direcciones: Dirección de Planificación para el Desarrollo, Dirección de Transformación del Estado, Dirección de Coordinación de Gobierno y Proyectos Especiales, con el objetivo de estipular y administrar las políticas públicas idóneas para beneficio de toda la comunidad salvadoreña.

Se han destaco estas cuatro instancias con miras, a analizar el por qué el resto de países centroamericanos como ser Panamá, Belice y Nicaragua no tomen a consideración la implementación de un órgano, dirección, secretaria donde se lleve un mejor manejo de las políticas públicas y así poder solventar un conflicto social que afecte a todos los sectores sociales. No solamente el Ministerio de Gobernación o Secretaria de Gobierno puede llevar a cabo la formulación como implementación de políticas públicas, tal vez parte de no contar con esta instancia no se puede obtener resultados adecuados o lo contrario, es por ello que obtienen resultados optimistas, depende de las personas encargadas en cada sector.

Para poder comenzar a comprender el panorama actual de las políticas públicas en materia de competitividad es importante abalizar el índice de competitividad de años más recientes 20152016. Según la Tabla 3, en Belice no se incluye en el Índice de Competitividad Global desde el 2013-2014 en adelante, por ello no se incluye. El país de la región que más ha mejorado significativamente ha sido Honduras y el Salvador. En el caso de la región centroamericana, dicho índice ha mostrado un comportamiento volátil y con muchas diferencias Panamá y Costa Rica se encuentran en las posiciones más altas en comparación a los países de la región, debido al mejoramiento global de sus economías. Sin embargo, han mostrado un relativo estancamiento debido a la caída de los precios de los productos que se comercializan, bajo niveles de comercio, inversión y ahorro, así como del bajo crecimiento de la productividad (Schwab, 2015). 
Tabla 3. Índice de Competitividad 2015-2017

\begin{tabular}{cccc}
\hline País & Índice 2015 (puntaje) & Índice 2016 & Posición 2017 ranking de 142 países \\
\hline Honduras & 3,82 & 3,95 & 96 \\
Guatemala & 4,1 & 4,05 & 84 \\
Costa Rica & 4,42 & 4,33 & 47 \\
El Salvador & 3,87 & 4,1 & 109 \\
Panamá & 4,43 & 4,38 & 42 \\
Nicaragua & 3,82 & 3,75 & 93 \\
\hline Elaboración propia con datos obtenidos World Economic Forum, The Global Competitiveness Report: \\
\multicolumn{4}{c}{ Schwab, K. (2015). }
\end{tabular}

En el caso de Honduras, se reconoce que, aunque ha mejorado significativamente, aún falta una política pública en competitividad que permita ser más asertiva y escalar más peldaños dentro del índice de competitividad. Por consiguiente, la necesidad de una política de competencia efectiva debe ser consistente dentro de los objetivos de política pública asociados con el mercado, dado que representa un pilar fundamental en el proceso de creación, asignación de riqueza y desarrollo de una economía. (Comisión para la Defensa y Promoción de la Competencia, 2015).

Ahora enfocando el tema de las políticas públicas en materia de competitividad, lo importante es que cada país maneja las políticas públicas ya se directamente en materia de competitividad como también en base a programas y planes.

En la tabla 4, se muestra un dato positivo en cuanto que los países centroamericanos si cuentan con políticas públicas con los 12 pilares de competitividad que exige el Foro Económico Mundial. Sin embargo, al analizar cada uno de los pilares persisten ciertas diferencias en cuanto a los países de la región. El primer pilar se encarga del Entorno Institucional, resumiendo en palabras claves, para las políticas públicas es que estas incluyan temas como ser la transparencia y lucha contra la corrupción.

Honduras cuenta con una Política Nacional de Transparencia y Acceso a la Información Pública (Miranda, 2015) . En los términos de referencia se establece las acciones que deben regir las instituciones tanto privadas como gubernamentales en el manejo de transparencia. Otro de los países que cuentan con políticas públicas en este primer pilar es Guatemala con la Política Nacional de Transparencia y lucha contra la corrupción.

De igual forma el Salvador cuenta con la Política de Transparencia Fiscal (Ministerio de Hacienda b, 2017). Dicha política determina las orientaciones, prioridades, estimación de la disponibilidad global de recursos y variables básicas para la asignación de los recursos; asimismo contiene de forma extractada las normas, métodos y procedimientos a los cuales deben darle cumplimiento las diferentes instituciones públicas en el proceso de elaboración de los proyectos de presupuesto institucional.

Finalmente, otro país centroamericano en contar con políticas públicas en esta área es Belice con su política de Acceso a la información y políticas de transparencia focalizada (Olabe \& Cruz, 2011). Un dato curioso es que para este pilar Costa Rica, Nicaragua y Panamá, no cuentan con una política pública directa, si no que va implícita dentro de su Plan de Gobierno. El porqué de ello se debe a que se gestiona dentro de un Plan operativo el manejo de las instituciones dentro de un plan concentrado de gobierno y no viendo como un ente alejado a la gestión gubernamental. 
Tabla 4. Cuadro comparativo de políticas de competitividad de Centroamérica

\begin{tabular}{|c|c|c|c|c|c|c|c|}
\hline Pilares & Guatemala & Salvador & Nicaragua & $\begin{array}{c}\text { Costa } \\
\text { Rica } \\
\end{array}$ & Honduras & Panamá & Belice \\
\hline Entorno Institucional: & $\mathrm{x}$ & $\mathrm{x}$ & $\mathrm{x}$ & $\mathrm{x}$ & $\mathrm{x}$ & $\mathrm{x}$ & $\mathrm{x}$ \\
\hline $\begin{array}{l}\text { Infraestructura amplia y } \\
\text { eficiente }\end{array}$ & $\mathrm{x}$ & $\mathrm{x}$ & $\mathrm{x}$ & $\mathrm{x}$ & $\mathrm{x}$ & $\mathrm{x}$ & $\mathrm{x}$ \\
\hline $\begin{array}{l}\text { Estabilidad } \\
\text { Macroeconómica }\end{array}$ & $\mathrm{x}$ & $\mathrm{x}$ & $\mathrm{x}$ & $\mathrm{x}$ & $\mathrm{x}$ & $\mathrm{x}$ & $\mathrm{x}$ \\
\hline $\begin{array}{l}\text { La salud y educación } \\
\text { primaria }\end{array}$ & $\mathrm{x}$ & $\mathrm{x}$ & $\mathrm{x}$ & $\mathrm{x}$ & $\mathrm{x}$ & $\mathrm{x}$ & $\mathrm{x}$ \\
\hline $\begin{array}{l}\text { A educación superior y } \\
\text { formación de la calidad } \\
\text { de educación superior }\end{array}$ & $\mathrm{x}$ & $\mathrm{x}$ & $\mathrm{x}$ & $\mathrm{x}$ & $\mathrm{x}$ & $\mathrm{x}$ & $\mathrm{x}$ \\
\hline $\begin{array}{l}\text { La eficiencia del } \\
\text { mercado de mercancías } \\
\text { en los países }\end{array}$ & $\mathrm{x}$ & $\mathrm{x}$ & $\mathrm{x}$ & $\mathrm{x}$ & $\mathrm{x}$ & $\mathrm{x}$ & $\mathrm{x}$ \\
\hline $\begin{array}{l}\text { La eficiencia del } \\
\text { mercado laboral }\end{array}$ & $\mathrm{x}$ & $\mathrm{x}$ & $\mathrm{x}$ & $\mathrm{x}$ & $\mathrm{x}$ & $\mathrm{x}$ & $\mathrm{X}$ \\
\hline $\begin{array}{l}\text { La sofisticación del } \\
\text { mercado financiero }\end{array}$ & $\mathrm{x}$ & $\mathrm{x}$ & $\mathrm{x}$ & $\mathrm{x}$ & $\mathrm{x}$ & $\mathrm{x}$ & $\mathrm{X}$ \\
\hline Disposición tecnológica & $\mathrm{x}$ & $\mathrm{x}$ & $\mathrm{x}$ & $\mathrm{x}$ & $\mathrm{x}$ & $\mathrm{x}$ & $\mathrm{X}$ \\
\hline El tamaño del mercado & $\mathrm{x}$ & $\mathrm{x}$ & $\mathrm{x}$ & $\mathrm{x}$ & $\mathrm{x}$ & $\mathrm{x}$ & $\mathrm{x}$ \\
\hline $\begin{array}{l}\text { Sofisticación de los } \\
\text { negocios }\end{array}$ & $\mathrm{x}$ & $\mathrm{x}$ & $\mathrm{x}$ & $\mathrm{x}$ & $\mathrm{x}$ & $\mathrm{x}$ & $\mathrm{x}$ \\
\hline Innovación & $x$ & $x$ & $\mathrm{x}$ & $x$ & $x$ & $\mathrm{X}$ & $x$ \\
\hline
\end{tabular}

Fuente: Elaboración propias con datos obtenidos de World Economic Forum, Agenda de Competitividad 2016

El segundo pilar que es en cuanto a infraestructura, enfocadoen calidad de redes de infraestructura en diversos lugares como escuelas, carreteras, edificios, transporte de mercancías. Al igual que el primer pilar se concentran en dos aspectos, en políticas públicas directas en el tema o concentradas en programas o planes de gobierno. En cuanto a Honduras, tiene una Política de Infraestructura analizada por el Banco Mundial como también el tema está presupuestado en el Plan de Gobierno 2010-2014 como en el actual Plan Nación (Plan de Gobierno , 2010)

Otro país que maneja este sistema dual en cuanto a políticas en infraestructura es Guatemala, donde tiene la Política General de Gobierno (2016-20121) el Plan Nacional de Infraestructura (Secretaría de Comunicación, 2016). Comparado con el Salvador, el cual es un país de extensión territorial relativamente pequeña, cuenta con una Política Nacional "Salvador Logístico” (Grupo LPG, 2017). Dicha política tiene como finalidad potencializar la infraestructura salvadoreña en cuanto a movilidad y transporte.

Para el caso de Costa Rica el manejo de la infraestructura se lleva a cabo por un Plan de Infraestructura 2015-2018, donde las políticas inmersas datan desde temas de presupuesto en infraestructura como también en lineamientos de las zonas más vulnerables tanto económicamente como estructuralmente (Escalante, 2014). Mientras que Nicaragua por su parte tiene Políticas y Proyectos de Desarrollo para Potenciar la Inversión 2017-2021 (Ramírez, 2016).

Dicho proyecto contiene Políticas y Proyectos de Desarrollo para aumentar el crecimiento y el desarrollo económico de Nicaragua en el siguiente período del Gobierno de Reconciliación y Unidad Nacional 2017-2021. En cuanto a Panamá y Belice, realizan un paréntesis en este pilar y no tiene políticas públicas directas, este tema esta enraizado en el Planes como el Plan Nacional de Turismo Sostenible para 2012-2030 de Belice y en Panamá el Plan de Gobierno 2015-2019 
El tercer pilar considerando hacer la fusión de los pilares Estabilidad Macroeconómica, Eficiencia de Mercado de Mercancías y Tamaño del Mercado cuyo tema principal es el enfoque económico para la competitividad. Dado que a que la mayoría de los países centroamericanos reducen el tema de economía como las políticas de su mercado en un solo plan o política que abarque dichos pilares para el índice de Competitividad Global. Con respecto a este pilar, los resultados son positivos. Todos los países centroamericanos cuentan con políticas públicas que abarcan los pilares fusionados con anterioridad.

Para destacar, Honduras cuenta con la Política Fiscal 2013-2016 al igual que el Plan Estratégico de Gobierno y el Plan 20/20 donde se establecen políticas para explotar el sector económico del país. Guatemala, Costa Rica, el Salvador, Panamá, Belice y Nicaragua también contienen políticas públicas en materia de competitividad económica en su Política Monetario donde especifican los sectores productivos del país como también políticas de desarrollo y potenciador en la económica nacional (Honduras 20/20, 2017)

En el cuarto pilar siendo este la Salud como pilar en competitividad, resalta la importancia de la labor gubernamental como de establecer mecanismos para que la sociedad se desarrolle tanto física y mentalmente (Politica Nacional Materno Infantil, 2015).Un punto interesante en la formulación de estas políticas públicas es la diferencia que se hace con respecto a que temas de salud se dará énfasis, por ejemplo, Honduras tiene políticas públicas en salud, pero enfocadas en Materno Infantil, Protección Social y Salud Alimentaria, considerando estos temas como en los que más necesita empoderarse el gobierno en la materia.

De la misma manera, Guatemala, cuenta con políticas públicas en salud pero enfocadas en temas más vulnerables como salud para las personas con enfermedades de transmisión sexual, esta política es la denominada : Política Pública Respecto a las Infecciones de Transmisión Sexual (ITS) y a la respuesta a la Epidemia del Síndrome de Inmunodeficiencia Adquirida (SIDA) (Ministerio de Salud Publica y Asistencia Social, 2015) .

Por su parte Costa Rica siendo de los países cuya investigación en materia de salud ha destacado no solo a nivel centroamericano si no a nivel latinoamericano, las políticas públicas actuales Seguridad Alimentaria y Nutricional 2011-2021, considerando por parte de sus gobiernos de turno en los cuales se necesita la mayor colaboración y gestión pública. En comparación con el resto de los países de la región, El Salvador, Nicaragua, Panamá y Belice que tiene una Política Nacional de Salud y para enfocarse en específico con otros temas que abarca la salud lo dividen en programas donde se ven inmersas políticas públicas como salud mental, protección social y nutrición (Plataforma de Seguridad Alimentaria y Nutricional, 2011).

El quinto pilar se enfoca en uno de los aspectos en los cuales los países centroamericanos se han visto en déficit como la Educación. Siendo la educación en los países centroamericanos está contemplada en tres niveles primordiales como la educación primaria, educación básica y educación superior. Para esta labor, cada Estado ha diseñado ciertas políticas públicas para cada una de las áreas anteriormente mencionadas, como el trabajo en conjunto con programas y planes.

Honduras cuenta con Política Nacional de Educación alternativa no formal , Educación Inicial y Pre-Básica, Educación Básica, Educación Media, Bono Vida Mejor que son políticas basadas en brindar un mejor educación en cada nivel y por edades a la población hondureña, sin embargo se cuenta con programas como ser la educación para las etnias donde se encuentran políticas inclusivas en materia de educación para estas minorías que necesitan de una tutela especial por parte del gobierno (Doryan, 1999). 
Al igual que Honduras, Guatemala maneja este mismo sistema en cuanto a dividir la educación, pero destaca con la política de educación inclusiva para la población con necesidades educativas especiales con y sin discapacidad, haciendo ese "agregado" en el cual no cuentan el resto de países de la región. También Costa Rica contiene políticas públicas divididas en cuanto al nivel educativo y según edad semejante a Honduras y Guatemala, pero contiene un aproximado de 12 programas encargados de incentivar la educación, siendo de estos programas los más mencionado Programa CEN CINAI y Programa Construyendo Alternativas Juveniles donde su principal objetivo es ayudar a los jóvenes costarricenses a que estudien ciertas carreras con el título de técnico pero considerando este como un rango alto y con fuente de trabajo en la sociedad.

De la misma forma, Panamá y Nicaragua contemplan políticas públicas en todos los nivele, sin embargo sus programas van más enraizados en cuanto a la educación de los más pequeños, como ser la política nicaragüense Política Nacional de Primera Infancia "Amor por los más chiquitos y chiquitas" y el de Panamá Proyecto de Alfabetización "Muévete por Panamá”. El único país en el cual no se encontró política pública en educación fue Belice, pero si con Programas en Educación de la UNESCO, siendo un trabajo en conjunto con el gobierno de Belice (Sistema de Información de Tendencias Educativas en America Latina, 2016).

El sexto pilar es en el ámbito de la eficiencia del mercado laboral, siendo de los más interesantes por la oferta de empleabilidad que cada país centroamericano maneja. En este pilar todos los países de la región utilizan políticas públicas. En el caso de Honduras se tiene 3 políticas Política integrada de empleo y protección social en Honduras, Política de Protección Social y Políticas de Empleo, Productividad y Salarios, las tres tiene el objetivo de disminuir los problemas constantes en encontrar un empleo como obtener un salario acorde a lo que la normatividad del país exige. Seguidamente Guatemala tiene 2 políticas públicas en materia de empleo Política Nacional de Empleo Digno 2017-2032 y Política Integrada de Comercio Exterior, Competitividad e Inversiones

Guatemala llama la atención por el hecho de incluir el tema de competitividad en la política de Comercio e Inversión como estrategia clave de generar empleo no solo por el gobierno si no de multinacionales como empresas público-privadas en l sociedad. Costa Rica por su parte tiene una política pública que concentra el tema de la empleabilidad, siendo la Política Nacional de Empleo, de la misma manera Nicaragua, El Salvador y Panamá contienen el mismo nombre de la política, pero resaltando el potencial de la inversión como fuente de generación de empleo. El caso de Belice no se encontró una política pública directa en el tema de empleo únicamente con un plan donde se reconoce ciertas políticas para el manejo de la empleabilidad como ser el Plan Sectorial de Desarrollo Social (Ministerio de Trabajo y Previsión Social, 2016).

El sétimo pilar es la sofisticación del mercado financiero, tanto en materia de inversiones como políticas encaminadas al desarrollo de la seguridad en inversiones. Para estas políticas, Honduras maneja la Política de Endeudamiento Público 2018-2021 y de la mano con el Plan Estratégico en Finanzas 2017-2020, cuyo objetivo es mejor la administración del gasto público como la administración financiera del Estado en particular y hacia donde son devengados los ingresos (Ministerio de Hacienda , 2012).

De la misma materia, Guatemala maneja el tema financiero en la Política Nacional de Desarrollo, viendo esta política la que contempla el sector económico y el financiero como instrumentos que deben llevarse de la mano (Banco Interamericano de Desarrollo, 2014).Similar a estos países anteriormente mencionados, Costa Rica tiene la Política de Inversión y de Finanzas como el Salvador con la Política de Inversión Pública, en las cuales se maneja el presupuesto financiero del Estado para los años del gobierno actual como también 
los mecanismos y acciones que se llevaran a cabo para mejorar la situación financiera del país mediante la inclusión de la sociedad civil (Hernandez, 2016)

Por su parte Nicaragua y Panamá en tema de finanzas utilizan políticas de desarrollo del Estado como Política Económica para manejar las finanzas del país por medio de políticas públicas que incluya la gestión de las finanzas y la económica. Nuevamente, no se encontró políticas públicas en cuanto al sector financiero por parte de Belice, únicamente un programa de finanzas rurales, donde se explica la necesidad del sector rural para a inversión y las finanzas, se deja a un lado el sector urbano en materia financiera (Público, 2016).

Finalmente, en el octavo pilar siendo la innovación y sofisticación de los negocios se resalta la importancia para los países centroamericanos de inmiscuirse en temas como ser nuevas tecnologías como el atractivo en cuando a los negocios que contenga. En el caso de Honduras no se cuenta con política pública en esta materia, únicamente programas. El Plan 20/20 como también el Plan Estratégico Regional de Ciencia, Tecnología e Innovación 2008-2018 (Junto con los miembros del SICA) donde se establecen los sectores en los que es básico poder implementar mecanismos de innovación como atractivo y ventaja competitividad (Bernal, 2008).

Guatemala tiene la Política Nacional de Desarrollo donde se aclara la importancia de la innovación no solo en los negocios si no como la modernización en técnicas de producción y de educación. Similar a Honduras, en Costa Rica no se encontró una política pública directa en materia de innovación, pero si un Plan Nacional de Ciencia, Tecnología e Innovación 20152021. En el Salvador y Panamá no se encontró tema de política pública en innovación pero si planes de desarrollo de ciencia, tecnología e innovación como base para desarrollar un mejor atractivo de negocios y uso de tecnologías que permitan más eficacia y reduzca los costos de producción, es decir, son planes vistos en materia económica (Comisión Económica para América Latina y el Caribe, 2010) .Caso particular, Belice, no se encontró política públicas ni programas como planes en este pilar.

Como se mencionó con anterioridad, Guatemala, cuenta con El Centro de Acopio de Políticas Públicas (2017) afirma las siguientes políticas:

- Agenda Nacional de Competitividad

- Política Nacional para el Desarrollo Turístico Sostenible de Guatemala 2012-2022

- Política Nacional de Producción más Limpia

Considerando contar con una estrategia clara en competitividad en comparación con el resto de los países centroamericanos. Estas políticas representan un panorama de los 12 pilares de competitividad del Foro Económico Mundial y como Guatemala realizará acciones para posicionarse en un índice de competitividad mejor cada año, comenzando desde el incremento de capital humano como el reconocimiento de la competitividad sistémica en este mundo globalizado en el que se habita.

\section{Conclusiones}

En los países de la región se puede hablar de la existencia de un mosaico de legislación, en general esta no ha nacido de una política coherente del gobierno. Como ejemplo de esto, es común que las políticas de un Ministerio o Secretaría de Estado sean contradictorias con lo ejecutado o previsto, ya que no siempre la acción gubernamental o la existencia de políticas públicas explícitas son requisitos para que la coordinación lleve a un buen equilibrio que refleje el nivel más alto posible de inversión. mediante la capacidad estratégica como ser una 
universidad, una institución multilateral, un gobierno foráneo, una amplia concertación nacional o un inversionista no residente.

En torno a la elaboración de políticas públicas se considera que, Nicaragua, aunque no sea altamente competitivo, tiene mucho que enseñar a sus vecinos, al igual que Honduras puede ofrecer algunas experiencias como Honduras y toda la región Centroamericana, como una experiencia de hermanos. En cuanto a la región centroamericana y su relación con el Salvador, se deben instalar conversaciones en temas de comercio y en qué medidas se puede desarrollar para ampliar las relaciones comerciales y también las relaciones de complementariedad productiva, como una región específicamente.

En cuanto a Panamá y Belice que en la mayoría de ocasiones se ve alejado del istmo centroamericano, han obtenido altos niveles en cuanto a competitividad si se compara con el resto de países centroamericanos. Este aspecto se debe, dado a que Panamá y Belice tiene políticas públicas enlazadas por medio de programas, planes y proyectos de desarrollo humano y estatal que permiten hacer de estos una zona atractiva para la comunidad internacional.

\section{Referencias bibliográficas}

Almond, G. A. (1978). Comparative Politics. System, Process and Policy. Boston: Little Brown.

Arias. (2006).

Astorga, C. R., \& Facio, M. A. (Septiembre de 2009). Eumed (Contibuciones a las Ciencias Sociales). Obtenido de ¿Qué son y para qué sirven las políticas públicas?: http://www.eumed.net/rev/cccss/05/aalf.htm

B.G, P. (1982). American Public Policy. Nueva York: Franklin Wats Pubs.

Banco Interamericano de Desarrollo. (2014). Panorama de las Administraciones Públicas. Obtenido de Ficha Actual el Salvador: https://www.oecd.org/gov/alc-el-salvador.pdf

Bejarano, J. A. (1997). Economía de la Agricultura. Colombia: Centro de Infromación y Documentación Rodrigo Peña.

Belize Ministry of Labor. (31 de Mayo de 2017). Ministry of Labor, Local Government and Rural Development, Public Service, Energy \& Public Utilities. Obtenido de República de Belice: http://www.publicservice.gov.bz/

Bernal, E. (2008). Plan estratégico regional de ciencia, tecnología e innovación 2008-2018. Obtenido de comisión para el desarrollo científico y tecnológico de Centroamérica, panama y republica dominicana: http://unpan1.un.org/intradoc/groups/public/documents/icap/unpan029739.pdf

Calderón Vásquez, F. J. (2015). EUMED (Enciclopedia Virtual). Obtenido de Las políticas publicas en la encrucijada: políticas sociales y competitividad sistémica: http://www.eumed.net/librosgratis/2008b/391/Politicas\%20Publicas\%20en\%20el\%20modelo\%20de\%20la\%20Co mpetitividad\%20Sistemica.htm 
Canto Sáenz, R. (12 de Marzo de 2014). Universidad Autónoma de Yucatán. Obtenido de Revista Perspectivas de Políticas Públicas: file://C:/Users/hp/Downloads/652-1370-1SM.pdf

Centro de Acopio de Políticas Públicas . (2017). Centro de Acopio de Políticas Públicas . Obtenido de Secretaria de Planificación y Programación SEGEPLAN: http://190.111.1.13/capp/?tag=competitividad

Chacón, C. M., \& Lawrence, P. (Septiembre de 1996). INCAE. Obtenido de Desarrollo Sostenible en Centroamérica: Políticas Públicas, Marco Legal e Institucional : http://www.incae.edu/ES/clacds/publicaciones/pdf/cen700filcorr.pdf

Chapa Gonzáles, H. M. (2010). ¿Cómo se mide la competitividad de las Naciones y que posición juega actualmente México? Punto de Vista (Universidad Nacional Autónoma de Chihuaha) , 1-5.

CO. (26 de Octubre de 2017). Plan estratégico regional de ciencia, tecnología e innovación 2008-2018. Obtenido de comisión para el desarrollo científico y tecnológico de Centroamerica: http://unpan1.un.org/intradoc/groups/public/documents/icap/unpan029739.pdf

Comisión Económica para América Latina y el Caribe. (Agosto de 2010). Plan nacional de ciencia, tecnología e innovación, Nicaragua 2010-2013. Obtenido de CEPAL: https://www.cepal.org/es/publicaciones/32845-plan-nacional-ciencia-tecnologiainnovacion-nicaragua-2010-2013

Comisión para la Defensa y Promoción de la Competencia. (16 de Noviembre de 2015). Honduras el país de Centroamérica que más ha mejorado su competitividad. Obtenido de https://www.cdpc.hn/?q=www.com_16112015

Delgado Godoy, L. (2009). Las políticas públicas. El ciclo de las políticas públicas. Clases de políticas públicas. Eficacia, legalidad y control. Indicadores de gestión. Gestión Administrativa, de la Administración de la Junta de Comunidades de Castilla-La Mancha , 1-15.

Doryan, E. (1999). Educación y Competitividad en Centroamerica . Obtenido de https://www.incae.edu/ES/clacds/publicaciones/pdf/cen1200filcorr.pdf

Escalante, A. C. (Noviembre de 2014). Plan Nacional de Desarrollo 2015-2018. Obtenido de https://documentos.mideplan.go.cr/alfresco/d/d/workspace/SpacesStore/cd1da1b4868b-4f6f-bdf8-b2dee0525b76/PND\%2020152018\%20Alberto\%20Ca\%C3\%B1as\%20Escalante\%20WEB.pdf

Fernández Arroyo, N. (Noviembre de 2012). UNICEF. Obtenido de CIPPEC: https://www.unicef.org/argentina/spanish/cippec_uni_planificacion.pdf

Forum, World Economic. (12 de Octubre de 2016). ¿Qué es la competitividad? Obtenido de https://www.weforum.org/es/agenda/2016/10/que-es-la-competitividad/

G.A, A., \& Powell, G. (1978). Comparative Politics. System, Process and Policy. Boston: Little Brown.

Gault, D. A., \& Blanco, F. (2013). Políticas públicas y democracia. Obtenido de Instituto Federal Electoral, Primera Edición: http://taoppcomunicacion.weebly.com/uploads/6/9/3/8/6938815/arellano_blanco_pol. _p\%C3\%B9bl._y_democracia.pdf 
Grupo LPG. (24 de Agosto de 2017). Presentan política nacional El Salvador Logístico. Obtenido de La Prensa Gráfica (Miembros de GDA, Grupo de Diarios America ): https://www.laprensagrafica.com/elsalvador/Presentan-politica-nacional-El-SalvadorLogistico-20170824-0083.html

Hernandez, O. (2016). Katun nuestra Guatemala 2032. Obtenido de Politica Nacional de Desarrollo : http://www.segeplan.gob.gt/downloads/2015/SPOT/Mandatos_y_Normativas/Politica s/Politica_Nacional_de_Desarrollo.pdf

Honduras 20/20. (2017). Honduras 20/20 Nuestra Visioón al Desarrollo. Obtenido de https://www.honduras2020.com/

Lahera, E. (Agosto de 2004). CEPAL. Obtenido de Política y Políticas Públicas: http://repositorio.cepal.org/bitstream/handle/11362/6085/S047600_es.pdf

Lahera, E. (Agosto de 2004). CEPAL. Obtenido de Política y Políticas Públicas: http://repositorio.cepal.org/bitstream/handle/11362/6085/S047600_es.pdf

Lahera, E. (Agosto de 2002). CEPAL, Fondo de Cultura Economica. Obtenido de Introducción a las Políticas Públicas: http://repositorio.cepal.org/bitstream/handle/11362/6085/S047600_es.pdf

López, D. (2009). Universidad Latina de América. Obtenido de ¿Qué es una política pública?: http://www.unla.mx/iusunla18/reflexion/QUE\%20ES\%20UNA\%20POLITICA\%20P UBLICA\%20web.htm

Malhotra, N. (2008). Investigacion de Mercados Quinta Edicion. En N. K. Malhotra, Metodologia de la investigacion (pág. 80). Mexico: Pearson Educacion.

Mendíaz, M. G. (2004). El estado y las políticas públicas las visiones desde neoinstitucionalismo. Obtenido de http://rephip.unr.edu.ar/bitstream/handle/2133/847/Mend\%C3\%ADaz_\%20El\%20Est ado\%20y\%20las\%20pol\%C3\%ADticas\%20p\%C3\%BAblicas_A1a.pdf?sequence=1

Mendoza Félix, J. C. (2015). Universidad Nacional de Piura. Obtenido de http://www.academia.edu/14497956/COMPETITIVIDAD_EMPRESARIAL

MiDEPLAN. (2016). MiDEPLAN, Rector de Sistema Nacional de Planificación. Obtenido de Ministerio de Planificación y Política Económica: http://www.mideplan.go.cr/

Ministerio de Gobernación. (2016). Ministerio de Gobernación. Obtenido de República de Nicaragua: https://www.migob.gob.ni/

Ministerio de Hacienda . (2012). Politica de Inversión Pública . Obtenido de Ministerio de Hacienda, Dirección General de Inversión y Crédito Público: http://www.transparenciafiscal.gob.sv/downloads/pdf/DC4065_Politica_de_Inversion. pdf_v0_0_1_31_08_2012.pdf

Ministerio de Hacienda b. (Marzo de 2017). Portal de Transparencia Fiscal. Obtenido de Políticas presupuestaria:

http://www.transparenciafiscal.gob.sv/ptf/es/PresupuestosPublicos/Polticaspresupuest arias.html 
Ministerio de Salud Publica y Asistencia Social. (2015). Política nacional de comadronas de los cuatro pueblos de guatemala 2015-2025. Obtenido de http://www.osarguatemala.org/osartemporal/Archivos/PDF/201604/312_2.pdf

Ministerio de Trabajo y Previsión Social. (18 de Diciembre de 2016). Polìtica Nacional de Empleo Digno 2017 - 2032. Obtenido de http://www.mintrabajo.gob.gt/index.php/98leyes-conveniosyacuerdos/6068-pol\%C3\%ACtica-nacional-de-empleo-digno-20172032.html

Miranda, E. (19 de Diciembre de 2015). Proyecto llevado a cabo por el Instituto de Acceso a la Información Pública. Obtenido de Instituto de acceso a la información pública Terminos de Referencia: http://www.honducompras.gob.hn/Docs/Lic563IAIP-CE-92014200-PliegooTerminosdeReferencia.pdf

Monge Pacheco, B. (20 de Septiembre de 2014). La República Premium. Obtenido de https://www.larepublica.net/noticia/_la_importancia_de_las_politicas_publicas_

Olabe, P. B., \& Cruz, J. (Noviembre de 2011). Sector de Capacidad Institucional y Finanzas. Obtenido de Banco Interamericano de Desarrollo: https://publications.iadb.org/bitstream/handle/11319/5350/Acceso\%20a\%20la\%20Info rmaci\%C3\%B3n\%20y\%20Pol\%C3\%ADticas\%20de\%20Transparencia\%20Focalizad a\%20\%20\%20\%20\%20.pdf?sequence=1

Público, C. d. (2016). Política de Endeudamiento Público 2018-2021. Obtenido de Taller Nacional de Estrategia de Financiamiento Sostenible: http://www.sefin.gob.hn/wpcontent/uploads/2017/03/PoliticaEndeudamientoPublico2018-2021.pdf

Parada, E. L. (2002). Introducción a las políticas públicas. Obtenido de Fondo de Cultura Económica: https://documentop.com/introduccion-a-las-politicaspublicas_59857eeb1723ddb404627f40.html

Pereyra, A. P. (2007). Políticas públicas para la promoción del desarrollo económico territorial. Quito: ASOCAM.

Peters, G. (1982). American Public Policy. Nueva York: Franklin Wats Pubs.

Plan de Gobierno . (2010). Plan de Gobierno 2010-2014 República de Honduras. Obtenido de http://www.redeplan.org/documents/10157/da90cced-9067-4ffd-a900-9e9d3f0116d5

Plataforma de Seguridad Alimentaria y Nutricional. (2011). Política nacional de seguridad alimentaria y nutricionaL 2011-2021. Obtenido de http://plataformacelac.org/politica/82

Plataformas para Informacion para Politicas Publicas . (25 de Septiembre de 2013). UNCUYO Universidad Nacional de Cuyo. Obtenido de Plataformas para Informacion para Politicas Publicas PiPP: http://www.politicaspublicas.uncu.edu.ar/novedad/loscaminos-para-la-competitividad

Politica Nacional Materno Infantil. (2015). Politica nacional materno infantil. Obtenido de Republica de Honduras: http://www.bvs.hn/E/pdf/PoliticaSMI.pdf

Quero, L. (2008). Estrategias competitivas: factor clave de desarrollo . Revista Científica Electrónica Ciencias Gerenciales (NEGOTIUM) , 1-14.

Ramírez, P. O. (3 de Octubre de 2016). Nicaragua presenta políticas y proyectos de desarrollo para potenciar la inversión en período 2017-2021. Obtenido de 
https://www.el19digital.com/articulos/ver/titulo:47248-nicaragua-presenta-politicas-yproyectos-de-desarrollo-para-potenciar-la-inversion-en-periodo-2017-2021.it

Schwab, K. (2015). World Economic Forum. Obtenido de The Global Competitiveness Report: http://www3.weforum.org/docs/gcr/20152016/Global_Competitiveness_Report_2015-2016.pdf

Secretaria de Comunicación del Estado. (2017). Secretaria de Comunicación del Estado. Obtenido de Republica de Guatemala: https://www.presidencia.gob.pa/

Secretaría de Comunicación. (2016). Politica General de Gobierno 2016-2020. Obtenido de Republica de Guatemala, Secretaría De Planificación Y Programación: http://www.segeplan.gob.gt/downloads/2016/PGG2016-2020.pdf

Secretaria General de Despacho de Gobierno. (2016). Dirección Presidencial de Planificación Estratégica, Presupuesto, Inversión Pública y Cooperación Externa. Obtenido de Secretaria General de Despacho de Gobierno, República de Honduras: http://www.scgg.gob.hn/content/planificacion-estrategica-presupuesto-e-inversionpublica

SEGEPLAN. (2016). Secretaria de Planificación y Progrmacion Republica de Guatemala. Obtenido de http://www.segeplan.gob.gt/nportal/

SETEPLAN. (2014). SETEPLAN presenta el subsistema de seguimiento y monitoreo del PQD 2014-2019. Obtenido de Secretaria Tecnica y de Planificación Republica de El Salvador: http://www.secretariatecnica.gob.sv/am_event/seteplan-presenta-elsubsistema-de-seguimiento-y-monitoreo-del-pqd-2014-2019/

Sistema de Información de Tendencias Educativas en America Latina. (2016). Sistema de Información de Tendencias Educativas en America Latina (SITEAL). Obtenido de http://www.siteal.iipe.unesco.org/politicas

Torres Melo, J., \& Santander, J. (2013). Introducción a las políticas públicas. Bogotá: IEMP.

Vázquez, F. J. (2016). Las políticas publicas en la encrucijada: políticas sociales y competitividad sistémica. Obtenido de Eumed (Enciclopedia Virtual): http://www.eumed.net/librosgratis/2008b/391/Politicas\%20Publicas\%20en\%20el\%20modelo\%20de\%20la\%20Co mpetitividad\%20Sistemica.htm 